\title{
Precise Computation of Energy Levels and Radiative Lifetimes in the $s, p, d$, and $f$ Sequence of Hydrogen Isotope, with Natural Line Widths
}

\author{
*Salman Raza ${ }^{1,2} \quad$ M. Adil Khan ${ }^{2,3} \quad$ Saqib Shahzad ${ }^{1} \quad$ Shahzaib Naeem ${ }^{1,4}$ \\ 1.Department of Physics, University of Karachi \\ 2.Department of Physics, Government National College No.1 \\ 3.Department of Physics, NED university of Engineering and Technology \\ 4.Department of Radiology, The Aga Khan University Hospital
}

\begin{abstract}
:
Energy levels and Radiative lifetimes in Deuterium for the following: $n s{ }^{2} S_{1 / 2}(n \geq 2), n p^{2} P^{o}(1 / 2,3 / 2)(n \geq 2)$, $n d$ ${ }^{2} D_{(3 / 2,5 / 2)}(n \geq 3)$, and $n f^{2} F^{o}(5 / 2,7 / 2)(n \geq 4)$ sequence have been evaluated with uncertainties in energies caused due to uncertainty principal. Theoretical calculations performed utilizing the Weakest Bound Electron Potential Model Theory (WBEPMT). Both sets of data show quite an excellent agreement with the experimental data listed at NIST. This theoretical computation is also a continuation of the work by Raza. S. et al. in Neutral Hydrogen. The high ' $n$ ' (principal quantum number) values for both sets of data are presented very first time by utilizing WBEPMT. Keywords: Energy levels, Radiative lifetimes, Quantum defects, Weakest bound electron, Natural line width.

DOI: $10.7176 /$ JNSR/9-10-07

Publication date:May $31^{\text {st }} 2019$

\section{Introduction}

Deuterium has many commercial and scientific applications. Like it is used as heavy water moderator in fission reactors, commonly used in nuclear magnetic resonance spectroscopy (NMR), in environmental sciences it is being used as a tracer, in nuclear weapons, and in drugs etc[1]. The need of its spectroscopic data is highly demanded. So, in our work we are going to utilize a computational method called WBEPMT for the computation of spectroscopic data of Deuterium [2]. Recently In 2018 Raza. S et.al. [3-4] computed the Rydberg energies series and radiative lifetimes of $\mathrm{H}$ I and In I by utilizing the same technique WBEPMT. And in past few years many elements in their atomic and ionic states were studied by this theory because of its semi-empirical nature. All these studies show remarkably good agreement in comparison with experimental results[5-22].

In this work first we obtained data from National Institute of Standards and Technology (NIST)[23] and rearranged them in an increasing order of quantum numbers ' $n$ ' and then by Utilizing the first few energy levels and radiative lifetimes of lowest ' $n$ ' values (experimental values of radiative lifetimes were obtained from transition probabilities listed at NIST) we computed energy levels sequence with quantum defects and radiative lifetimes with natural line widths for the $s, p, d$ and $f$ sequence of Deuterium up to $n=60$. Both sets of data show excellent agreement with experimental data [23].
\end{abstract}

\section{Theory and Computational Approach:}

The WBEPMT used is very effective and simplest model for calculating energy level sequence of Rydberg atoms and ions. Unlike previously published theoretical methods, it relies on a simple concept of non-weakest bound electrons (NWBE's) and weakest bound electron (WBE). The ionic-core of $+(Z-1)$ charge formed with nucleus and NWBE's. The WBE moves under the influence of effective potential of an ion-core. During consecutive ionization step by step all WBE's separates from the ion-core, in each step only one weakest bound electron (WBE) ionize and rest non-weakest bound electrons (NWBE's) forming a new ion-core with effective potential ( the effective potential of ion-core is due to penetration, polarization and shielding effect). This effective potential in which WBE is residing can be given as[2]:

$$
\begin{gathered}
V(r)=-\frac{Z}{r}+\frac{Y}{r^{2}} \\
\text { where, } Y=\frac{m(m+1)+2 m l}{2}
\end{gathered}
$$

In equation (1) the first and second terms at right hand side represent coulombic potential and polarization potential respectively. The polarization potential created by the dipole formed between ion-core and WBE.

In first term, the separation distance between nucleus and WBE's is ' $r$ ', the effective nucleus charge is $Z$ and in $\mathrm{Y}$, the angular quantum number of WBE's is ' $l$ ', and $\mathrm{m}$ is an unknown coefficient not necessarily an integer.

So, the energy formula for WBE's is:

$$
T=-\frac{1}{2}\left(\frac{Z^{*}}{n^{*}}\right)^{2}
$$

In equation (2), $n^{*}$ is the effective quantum number, $n^{*}$ and $Z^{*}$ are unknown parameters, but the problem is solved 
by the transformation between Eigen-values of Quantum defect theory (QDT) and WBE potential model theory, given as:

$$
\frac{Z^{*}}{n^{*}}=\frac{Z_{o}}{n-\delta_{n}}
$$

In equation (3) $Z_{o}$ is the atomic kernel net charge number $\left(Z_{o}=1,2\right.$, and $3 \ldots$ for the ion-core charge in (QDT) and $\delta_{n}$ is the quantum defect of nth energy level.

Now, the energy levels of Rydberg states of an atomic system in the WBEPMT written as: [24]

$$
E=T_{\text {lim }}+T
$$

In equation (4) the first term in right hand side is the ionization limit $T_{\text {lim }}$ and $2^{\text {nd }}$ term is the energy $T$ of WBE's.

Now, by combining equations (3) and (4), we can rewrite (4) as:

$$
E=T_{\text {lim }}-\frac{1}{2}\left(\frac{Z_{o}}{n-\delta_{n}}\right)^{2}
$$

The quantum defect ' $\delta_{n}$ ' in equation (5) are computed by using Martin's formula for energy levels sequence, can be given as: [25].

$$
\delta_{n}=a_{0}+a_{1}\left(n-\delta_{0}\right)^{-2}+a_{2}\left(n-\delta_{0}\right)^{-4}+a_{3}\left(n-\delta_{0}\right)^{-6}
$$

In equation (6) $\delta_{0}$ is the lowest energy level quantum defect of the series, coefficients $\left(a_{i}\right.$ 's, $\left.\mathrm{i}=0,1,2,3\right)$ in $(6)$ are obtained by the method of least-square fitting, by using the first few given experimental values of the energy levels sequence. [26].

By utilizing the Quantum defects of energy levels sequence, the radiative lifetimes of the energy levels can be found by Rykova's expression [27].

In which

$$
\tau=\tau_{0}(n *)^{\alpha}
$$

In equation (9), $\tau_{o}$ and $\alpha$ are the parameter of Rykova's expression. A least square method can be used to calculate values of the parameters with the help of known radiative lifetimes of first few levels.

utilizing The Radiative lifetimes ' $\tau$ ', the uncertainty in energy levels due to principal of uncertainty can be computed by using expression [28].

$$
\Gamma=\frac{h}{2 \Pi \tau}
$$

In equation (10), $\Gamma$ is natural line width and $\mathrm{h}$ is Planck's constant.

\section{Result and Discussion:}

\subsection{Energy Levels Sequence with Quantum defects:}

In this work first we computed energy levels sequence with quantum defects for the following: $n s{ }^{2} S_{1 / 2}(n \geq 2)$, $n p^{2} P_{(1 / 2,3 / 2)}^{o}(n \geq 2)$, $n d^{2} D_{(3 / 2,5 / 2)}(n \geq 3)$, and $n f^{2} F^{o}(5 / 2,7 / 2)(n \geq 4)$ sequence of deuterium. For the quantum defects are computed by equation 6 in text (Martin's expression) for $s, p, d$ and $f$ sequence. The coefficients of equation 6 are listed in Table I: computed by first few experimental values of energy levels obtained from NIST[23].

Table I. Shows the Coefficients of Martin's Expression \& Sequence Convergence ( Limit:109708.61455299 $\mathrm{cm}^{-}$ 1)

\begin{tabular}{lccccccc}
\hline \hline Energy Sequence & $a_{0}$ & $a_{1}$ & $a_{2}$ & $a_{3}$ & $\delta_{0}$ & $\delta_{60}$ & Convergence Nature \\
\hline$n s^{2} S_{I / 2}(n \geq 2)$ & -0.00105 & 0.01291 & -0.08122 & 0.16919 & -0.00025 & -0.00105 & Core-polarization \\
$n P^{2} P^{o} 1 / 2(n \geq 2)$ & -0.00106 & 0.01291 & -0.08121 & 0.16919 & -0.00027 & -0.00106 & Core-polarization \\
$n P^{2} P^{o}{ }_{3 / 2}(n \geq 2)$ & -0.00105 & 0.01291 & -0.08121 & 0.16918 & -0.00025 & -0.00104 & Core-polarization \\
$n d^{2} D_{3 / 2}(n \geq 3)$ & -0.00135 & 0.02716 & -0.30029 & 1.19460 & -0.00040 & -0.00134 & Core-polarization \\
$n d^{2} D_{5 / 2}(n \geq 3)$ & -0.00135 & 0.02716 & -0.30029 & 1.19459 & -0.00040 & -0.00134 & Core-polarization \\
$n f^{2} F^{o} 5 / 2(n \geq 4)$ & -0.00127 & 0.02079 & -0.14486 & 0.00000 & -0.00054 & -0.00126 & Core-polarization \\
$n f^{2} F^{o} 7 / 2(n \geq 4)$ & -0.00127 & 0.02079 & -0.14486 & 0.00000 & -0.00054 & -0.00127 & Core-polarization \\
\hline \hline
\end{tabular}

From above Table I. we can clearly see by the comparison of ' $\delta_{60}$ ' the quantum defects of the highest energy level up to which energy level sequence computed in this work with ' $\mathrm{a}_{0}$ 'the first coefficient of equation 6 in text, $s, p, d$ and $f$ sequence of quantum defects converge toward ' $a_{0}$ '. The negative sign of ' $a_{0}$ ' indicates that corepolarization potential dominates the ' $n l$ ' $(l=s, p, d$ and $f)$ energy levels sequence.

Using these quantum defects energy levels sequence of all ' $n l$ ' sequence of Deuterium are determined precisely by utilizing equation 4 in text up to $n=60$ principal quantum number, listed in Tables: II-VIII. The results are in good accuracy with NIST values[23]. The energy levels and Quantum Defects for high ' $n$ ' values are presented very first time. 
Table II: Energy Level sequence in $\mathrm{cm}^{-1}$ and Quantum Defects of $n s^{2} S_{1 / 2}(2 \leq n \leq 60)$ in Deuterium.

\begin{tabular}{|c|c|c|c|c|c|c|c|c|c|}
\hline \multirow{2}{*}{$n$} & \multicolumn{2}{|c|}{ Quantum defects } & \multicolumn{2}{|c|}{ Energy Levels } & \multirow[b]{2}{*}{$n$} & \multicolumn{2}{|c|}{ Quantum defects } & \multicolumn{2}{|c|}{ Energy Levels } \\
\hline & 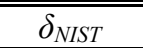 & $\delta_{C a l}$ & $\overline{E_{N I S T}}$ & $E_{\text {Cal }}$ & & $\overline{\delta_{\text {NIST }}}$ & $\delta_{\text {Cal }}$ & $\overline{E_{\text {NIST }}}$ & $E_{C a l}$ \\
\hline 2 & -0.00025 & -0.00025 & 82281.34 & 82281.34 & 32 & & -0.00104 & & 109601.46 \\
\hline 3 & -0.00039 & -0.00039 & 97518.75 & 97518.75 & 33 & & -0.00104 & & 109607.85 \\
\hline 4 & -0.00052 & -0.00052 & 102851.83 & 102851.83 & 34 & & -0.00104 & & 109613.69 \\
\hline 5 & -0.00065 & -0.00065 & 105320.28 & 105320.28 & 35 & & -0.00104 & & 109619.04 \\
\hline 6 & -0.00079 & -0.00075 & 106661.16 & 106661.13 & 36 & & -0.00104 & & 109623.95 \\
\hline 7 & -0.00092 & -0.00082 & 107469.67 & 107469.61 & 37 & & -0.00104 & & 109628.46 \\
\hline 8 & -0.00106 & -0.00087 & 107994.43 & 107994.35 & 38 & & -0.00104 & & 109632.62 \\
\hline 9 & -0.00119 & -0.00090 & 108354.19 & 108354.11 & 39 & & -0.00104 & & 109636.47 \\
\hline 10 & -0.00132 & -0.00093 & 108611.54 & 108611.45 & 40 & & -0.00104 & & 109640.03 \\
\hline 11 & -0.00146 & -0.00095 & 108801.94 & 108801.85 & 41 & & -0.00104 & & 109643.34 \\
\hline 12 & -0.00159 & -0.00096 & 108946.75 & 108946.67 & 42 & & -0.00104 & & 109646.41 \\
\hline 13 & & -0.00098 & & 109059.38 & 43 & & -0.00104 & & 109649.27 \\
\hline 14 & & -0.00099 & & 109148.81 & 44 & & -0.00104 & & 109651.93 \\
\hline 15 & & -0.00099 & & 109220.96 & 45 & & -0.00104 & & 109654.43 \\
\hline 16 & & -0.00100 & & 109280.01 & 46 & & -0.00104 & & 109656.76 \\
\hline 17 & & -0.00101 & & 109328.95 & 47 & & -0.00104 & & 109658.94 \\
\hline 18 & & -0.00101 & & 109369.96 & 48 & & -0.00104 & & 109660.99 \\
\hline 19 & & -0.00101 & & 109404.67 & 49 & & -0.00104 & & 109662.91 \\
\hline 20 & & -0.00102 & & 109434.30 & 50 & & -0.00104 & & 109664.72 \\
\hline 21 & & -0.00102 & & 109459.80 & 51 & & -0.00104 & & 109666.43 \\
\hline 22 & & -0.00102 & & 109481.91 & 52 & & -0.00105 & & 109668.03 \\
\hline 23 & & -0.00103 & & 109501.19 & 53 & & -0.00105 & & 109669.55 \\
\hline 24 & & -0.00103 & & 109518.12 & 54 & & -0.00105 & & 109670.98 \\
\hline 25 & & -0.00103 & & 109533.05 & 55 & & -0.00105 & & 109672.34 \\
\hline 26 & & -0.00103 & & 109546.29 & 56 & & -0.00105 & & 109673.62 \\
\hline 27 & & -0.00103 & & 109558.10 & 57 & & -0.00105 & & 109674.84 \\
\hline 28 & & -0.00103 & & 109568.65 & 58 & & -0.00105 & & 109675.99 \\
\hline 29 & & -0.00103 & & 109578.14 & 59 & & -0.00105 & & 109677.09 \\
\hline 30 & & -0.00104 & & 109586.69 & 60 & & -0.00105 & & 109678.13 \\
\hline 31 & & -0.00104 & & 109594.43 & & & & & \\
\hline
\end{tabular}


Table III: Energy Level sequence in $\mathrm{cm}^{-1}$ and Quantum Defects of $n p^{2} P_{1 / 2}^{o}(2 \leq n \leq 60)$ in Deuterium.

\begin{tabular}{|c|c|c|c|c|c|c|c|c|c|}
\hline \multirow{2}{*}{$n$} & \multicolumn{2}{|c|}{ Quantum defects } & \multicolumn{2}{|c|}{ Energy Levels } & \multirow{2}{*}{$n$} & \multicolumn{2}{|c|}{ Quantum defects } & \multicolumn{2}{|c|}{ Energy Levels } \\
\hline & $\delta_{N I S T}$ & $\delta_{\text {Cal }}$ & $E_{N I S T}$ & $E_{C a l}$ & & $\delta_{N I S T}$ & $\delta_{\text {Cal }}$ & $E_{N I S T}$ & $E_{C a l}$ \\
\hline 2 & -0.00025 & -0.00025 & 82281.30 & 82281.30 & 32 & & -0.00104 & & 109601.46 \\
\hline 3 & -0.00038 & -0.00038 & 97518.74 & 97518.74 & 33 & & -0.00104 & & 109607.85 \\
\hline 4 & -0.00052 & -0.00052 & 102851.83 & 102851.83 & 34 & & -0.00104 & & 109613.69 \\
\hline 5 & -0.00065 & -0.00065 & 105320.28 & 105320.28 & 35 & & -0.00104 & & 109619.04 \\
\hline 6 & -0.00079 & -0.00075 & 106661.16 & 106661.13 & 36 & & -0.00104 & & 109623.95 \\
\hline 7 & -0.00092 & -0.00082 & 107469.67 & 107469.61 & 37 & & -0.00104 & & 109628.46 \\
\hline 8 & -0.00105 & -0.00087 & 107994.43 & 107994.35 & 38 & & -0.00104 & & 109632.62 \\
\hline 9 & -0.00119 & -0.00090 & 108354.19 & 108354.11 & 39 & & -0.00104 & & 109636.47 \\
\hline 10 & -0.00132 & -0.00093 & 108611.53 & 108611.45 & 40 & & -0.00104 & & 109640.03 \\
\hline 11 & -0.00146 & -0.00095 & 108801.94 & 108801.85 & 41 & & -0.00104 & & 109643.34 \\
\hline 12 & -0.00159 & -0.00096 & 108946.75 & 108946.67 & 42 & & -0.00104 & & 109646.41 \\
\hline 13 & & -0.00097 & & 109059.38 & 43 & & -0.00104 & & 109649.27 \\
\hline 14 & & -0.00098 & & 109148.81 & 44 & & -0.00104 & & 109651.93 \\
\hline 15 & & -0.00099 & & 109220.96 & 45 & & -0.00104 & & 109654.43 \\
\hline 16 & & -0.00100 & & 109280.01 & 46 & & -0.00104 & & 109656.76 \\
\hline 17 & & -0.00100 & & 109328.95 & 47 & & -0.00104 & & 109658.94 \\
\hline 18 & & -0.00101 & & 109369.96 & 48 & & -0.00104 & & 109660.99 \\
\hline 19 & & -0.00101 & & 109404.67 & 49 & & -0.00104 & & 109662.91 \\
\hline 20 & & -0.00102 & & 109434.30 & 50 & & -0.00104 & & 109664.72 \\
\hline 21 & & -0.00102 & & 109459.80 & 51 & & -0.00104 & & 109666.43 \\
\hline 22 & & -0.00102 & & 109481.91 & 52 & & -0.00104 & & 109668.03 \\
\hline 23 & & -0.00102 & & 109501.19 & 53 & & -0.00104 & & 109669.55 \\
\hline 24 & & -0.00103 & & 109518.12 & 54 & & -0.00104 & & 109670.98 \\
\hline 25 & & -0.00103 & & 109533.05 & 55 & & -0.00104 & & 109672.34 \\
\hline 26 & & -0.00103 & & 109546.29 & 56 & & -0.00104 & & 109673.62 \\
\hline 27 & & -0.00103 & & 109558.10 & 57 & & -0.00104 & & 109674.84 \\
\hline 28 & & -0.00103 & & 109568.65 & 58 & & -0.00104 & & 109675.99 \\
\hline 29 & & -0.00103 & & 109578.14 & 59 & & -0.00104 & & 109677.09 \\
\hline 30 & & -0.00103 & & 109586.69 & 60 & & -0.00104 & & 109678.13 \\
\hline 31 & & -0.00104 & & 109594.43 & & & & & \\
\hline
\end{tabular}


Table IV: Energy Level sequence in $\mathrm{cm}^{-1}$ and Quantum Defects of $n d^{2} P^{o}{ }_{3 / 2}(2 \leq n \leq 60)$ in Deuterium.

\begin{tabular}{|c|c|c|c|c|c|c|c|c|c|}
\hline \multirow{2}{*}{$n$} & \multicolumn{2}{|c|}{ Quantum defects } & \multicolumn{2}{|c|}{ Energy Levels } & \multirow{2}{*}{$n$} & \multicolumn{2}{|c|}{ Quantum defects } & \multicolumn{2}{|c|}{ Energy Levels } \\
\hline & 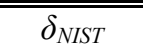 & $\delta_{\mathrm{Cal}}$ & $\overline{E_{N I S T}}$ & $\overline{E_{C a l}}$ & & " & $\delta_{\text {Cal }}$ & 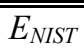 & $\overline{E_{\mathrm{Cal}}}$ \\
\hline 2 & -0.00027 & -0.00027 & 82281.67 & 82281.67 & 32 & & -0.00105 & & 109601.46 \\
\hline 3 & -0.00040 & -0.00040 & 97518.85 & 97518.85 & 33 & & -0.00105 & & 109607.85 \\
\hline 4 & -0.00053 & -0.00053 & 102851.87 & 102851.87 & 34 & & -0.00105 & & 109613.69 \\
\hline 5 & -0.00066 & -0.00066 & 105320.30 & 105320.30 & 35 & & -0.00105 & & 109619.04 \\
\hline 6 & -0.00080 & -0.00076 & 106661.18 & 106661.14 & 36 & & -0.00105 & & 109623.95 \\
\hline 7 & -0.00093 & -0.00083 & 107469.68 & 107469.62 & 37 & & -0.00105 & & 109628.46 \\
\hline 8 & -0.00107 & -0.00088 & 107994.43 & 107994.35 & 38 & & -0.00105 & & 109632.62 \\
\hline 9 & -0.00120 & -0.00091 & 108354.20 & 108354.11 & 39 & & -0.00105 & & 109636.47 \\
\hline 10 & -0.00134 & -0.00094 & 108611.54 & 108611.45 & 40 & & -0.00105 & & 109640.03 \\
\hline 11 & -0.00147 & -0.00096 & 108801.94 & 108801.86 & 41 & & -0.00105 & & 109643.34 \\
\hline 12 & -0.00161 & -0.00098 & 108946.76 & 108946.68 & 42 & & -0.00105 & & 109646.41 \\
\hline 13 & & -0.00099 & & 109059.38 & 43 & & -0.00105 & & 109649.27 \\
\hline 14 & & -0.00100 & & 109148.81 & 44 & & -0.00106 & & 109651.93 \\
\hline 15 & & -0.00101 & & 109220.96 & 45 & & -0.00106 & & 109654.43 \\
\hline 16 & & -0.00101 & & 109280.01 & 46 & & -0.00106 & & 109656.76 \\
\hline 17 & & -0.00102 & & 109328.95 & 47 & & -0.00106 & & 109658.94 \\
\hline 18 & & -0.00102 & & 109369.96 & 48 & & -0.00106 & & 109660.99 \\
\hline 19 & & -0.00103 & & 109404.67 & 49 & & -0.00106 & & 109662.91 \\
\hline 20 & & -0.00103 & & 109434.30 & 50 & & -0.00106 & & 109664.72 \\
\hline 21 & & -0.00103 & & 109459.80 & 51 & & -0.00106 & & 109666.43 \\
\hline 22 & & -0.00104 & & 109481.91 & 52 & & -0.00106 & & 109668.03 \\
\hline 23 & & -0.00104 & & 109501.19 & 53 & & -0.00106 & & 109669.55 \\
\hline 24 & & -0.00104 & & 109518.12 & 54 & & -0.00106 & & 109670.98 \\
\hline 25 & & -0.00104 & & 109533.05 & 55 & & -0.00106 & & 109672.34 \\
\hline 26 & & -0.00104 & & 109546.29 & 56 & & -0.00106 & & 109673.62 \\
\hline 27 & & -0.00104 & & 109558.10 & 57 & & -0.00106 & & 109674.84 \\
\hline 28 & & -0.00105 & & 109568.65 & 58 & & -0.00106 & & 109675.99 \\
\hline 29 & & -0.00105 & & 109578.14 & 59 & & -0.00106 & & 109677.09 \\
\hline 30 & & -0.00105 & & 109586.69 & 60 & & -0.00106 & & 109678.13 \\
\hline 31 & & -0.00105 & & 109594.43 & & & & & \\
\hline
\end{tabular}


Table V: Energy Level Sequence in $\mathrm{cm}^{-1}$ and Quantum Defects of $n d^{2} D_{3 / 2}(3 \leq n \leq 60)$ in Deuterium.

\begin{tabular}{|c|c|c|c|c|c|c|c|c|c|}
\hline \multirow{2}{*}{$n$} & \multicolumn{2}{|c|}{ Quantum defects } & \multicolumn{2}{|c|}{ Energy Levels } & \multirow{2}{*}{$n$} & \multicolumn{2}{|c|}{ Quantum defects } & \multicolumn{2}{|c|}{ Energy Levels } \\
\hline & $\delta_{N I S T}$ & $\delta_{\mathrm{Cal}}$ & $E_{N I S T}$ & $E_{C a l}$ & & $\delta_{N I S T}$ & $\delta_{C a l}$ & $E_{N I S T}$ & $E_{C a l}$ \\
\hline 3 & -0.00040 & -0.00040 & 97518.85 & 97518.85 & 32 & & -0.00132 & & 109601.46 \\
\hline 4 & -0.00053 & -0.00053 & 102851.87 & 102851.87 & 33 & & -0.00132 & & 109607.85 \\
\hline 5 & -0.00066 & -0.00066 & 105320.30 & 105320.30 & 34 & & -0.00132 & & 109613.69 \\
\hline 6 & -0.00080 & -0.00080 & 106661.18 & 106661.18 & 35 & & -0.00133 & & 109619.04 \\
\hline 7 & -0.00093 & -0.00091 & 107469.68 & 107469.66 & 36 & & -0.00133 & & 109623.95 \\
\hline 8 & -0.00107 & -0.00099 & 107994.43 & 107994.40 & 37 & & -0.00133 & & 109628.46 \\
\hline 9 & -0.00120 & -0.00106 & 108354.20 & 108354.15 & 38 & & -0.00133 & & 109632.62 \\
\hline 10 & -0.00134 & -0.00110 & 108611.54 & 108611.49 & 39 & & -0.00133 & & 109636.47 \\
\hline 11 & -0.00147 & -0.00114 & 108801.94 & 108801.89 & 40 & & -0.00133 & & 109640.03 \\
\hline 12 & -0.00161 & -0.00117 & 108946.76 & 108946.70 & 41 & & -0.00133 & & 109643.34 \\
\hline 13 & & -0.00120 & & 109059.40 & 42 & & -0.00133 & & 109646.41 \\
\hline 14 & & -0.00122 & & 109148.83 & 43 & & -0.00133 & & 109649.27 \\
\hline 15 & & -0.00123 & & 109220.97 & 44 & & -0.00133 & & 109651.94 \\
\hline 16 & & -0.00125 & & 109280.02 & 45 & & -0.00133 & & 109654.43 \\
\hline 17 & & -0.00126 & & 109328.96 & 46 & & -0.00133 & & 109656.76 \\
\hline 18 & & -0.00127 & & 109369.97 & 47 & & -0.00133 & & 109658.94 \\
\hline 19 & & -0.00127 & & 109404.67 & 48 & & -0.00134 & & 109660.99 \\
\hline 20 & & -0.00128 & & 109434.31 & 49 & & -0.00134 & & 109662.91 \\
\hline 21 & & -0.00129 & & 109459.81 & 50 & & -0.00134 & & 109664.72 \\
\hline 22 & & -0.00129 & & 109481.91 & 51 & & -0.00134 & & 109666.43 \\
\hline 23 & & -0.00130 & & 109501.20 & 52 & & -0.00134 & & 109668.03 \\
\hline 24 & & -0.00130 & & 109518.12 & 53 & & -0.00134 & & 109669.55 \\
\hline 25 & & -0.00130 & & 109533.05 & 54 & & -0.00134 & & 109670.98 \\
\hline 26 & & -0.00131 & & 109546.30 & 55 & & -0.00134 & & 109672.34 \\
\hline 27 & & -0.00131 & & 109558.10 & 56 & & -0.00134 & & 109673.62 \\
\hline 28 & & -0.00131 & & 109568.66 & 57 & & -0.00134 & & 109674.84 \\
\hline 29 & & -0.00132 & & 109578.14 & 58 & & -0.00134 & & 109676.00 \\
\hline 30 & & -0.00132 & & 109586.70 & 59 & & -0.00134 & & 109677.09 \\
\hline 31 & & -0.00132 & & 109594.43 & 60 & & -0.00134 & & 109678.13 \\
\hline
\end{tabular}


Table VI: Energy Level Sequence in $\mathrm{cm}^{-1}$ and Quantum Defects of $n d^{2} D_{5 / 2}(3 \leq n \leq 60)$ in Deuterium.

\begin{tabular}{|c|c|c|c|c|c|c|c|c|c|}
\hline \multirow{2}{*}{$n$} & \multicolumn{2}{|c|}{ Quantum defects } & \multicolumn{2}{|c|}{ Energy Levels } & \multirow{2}{*}{$n$} & \multicolumn{2}{|c|}{ Quantum defects } & \multicolumn{2}{|c|}{ Energy Levels } \\
\hline & $\delta_{N I S T}$ & $\delta_{\mathrm{Cal}}$ & $E_{N I S T}$ & $E_{C a l}$ & & $\delta_{N I S T}$ & $\delta_{C a l}$ & $E_{N I S T}$ & $E_{C a l}$ \\
\hline 3 & -0.00040 & -0.00040 & 97518.88 & 97518.88 & 32 & & -0.00133 & & 109601.46 \\
\hline 4 & -0.00054 & -0.00054 & 102851.89 & 102851.89 & 33 & & -0.00133 & & 109607.85 \\
\hline 5 & -0.00067 & -0.00067 & 105320.31 & 105320.31 & 34 & & -0.00133 & & 109613.69 \\
\hline 6 & -0.00080 & -0.00080 & 106661.18 & 106661.18 & 35 & & -0.00133 & & 109619.04 \\
\hline 7 & -0.00094 & -0.00091 & 107469.68 & 107469.67 & 36 & & -0.00133 & & 109623.95 \\
\hline 8 & -0.00107 & -0.00100 & 107994.43 & 107994.40 & 37 & & -0.00133 & & 109628.46 \\
\hline 9 & -0.00121 & -0.00106 & 108354.20 & 108354.16 & 38 & & -0.00133 & & 109632.62 \\
\hline 10 & -0.00134 & -0.00111 & 108611.54 & 108611.49 & 39 & & -0.00133 & & 109636.47 \\
\hline 11 & -0.00148 & -0.00115 & 108801.94 & 108801.89 & 40 & & -0.00133 & & 109640.03 \\
\hline 12 & -0.00161 & -0.00118 & 108946.76 & 108946.70 & 41 & & -0.00134 & & 109643.34 \\
\hline 13 & & -0.00120 & & 109059.40 & 42 & & -0.00134 & & 109646.41 \\
\hline 14 & & -0.00122 & & 109148.83 & 43 & & -0.00134 & & 109649.27 \\
\hline 15 & & -0.00124 & & 109220.97 & 44 & & -0.00134 & & 109651.94 \\
\hline 16 & & -0.00125 & & 109280.02 & 45 & & -0.00134 & & 109654.43 \\
\hline 17 & & -0.00126 & & 109328.96 & 46 & & -0.00134 & & 109656.76 \\
\hline 18 & & -0.00127 & & 109369.97 & 47 & & -0.00134 & & 109658.94 \\
\hline 19 & & -0.00128 & & 109404.67 & 48 & & -0.00134 & & 109660.99 \\
\hline 20 & & -0.00129 & & 109434.31 & 49 & & -0.00134 & & 109662.91 \\
\hline 21 & & -0.00129 & & 109459.81 & 50 & & -0.00134 & & 109664.72 \\
\hline 22 & & -0.00130 & & 109481.91 & 51 & & -0.00134 & & 109666.43 \\
\hline 23 & & -0.00130 & & 109501.20 & 52 & & -0.00134 & & 109668.03 \\
\hline 24 & & -0.00131 & & 109518.12 & 53 & & -0.00134 & & 109669.55 \\
\hline 25 & & -0.00131 & & 109533.05 & 54 & & -0.00134 & & 109670.98 \\
\hline 26 & & -0.00131 & & 109546.30 & 55 & & -0.00134 & & 109672.34 \\
\hline 27 & & -0.00131 & & 109558.10 & 56 & & -0.00134 & & 109673.62 \\
\hline 28 & & -0.00132 & & 109568.66 & 57 & & -0.00134 & & 109674.84 \\
\hline 29 & & -0.00132 & & 109578.14 & 58 & & -0.00134 & & 109676.00 \\
\hline 30 & & -0.00132 & & 109586.70 & 59 & & -0.00134 & & 109677.09 \\
\hline 31 & & -0.00132 & & 109594.43 & 60 & & -0.00134 & & 109678.13 \\
\hline
\end{tabular}


Table VII: Energy Level Sequence in $\mathrm{cm}^{-1}$ and Quantum Defects of $n f^{2} F^{o}{ }_{5 / 2}(4 \leq n \leq 60)$ in Deuterium.

\begin{tabular}{|c|c|c|c|c|c|c|c|c|c|}
\hline \multirow{2}{*}{$n$} & \multicolumn{2}{|c|}{ Quantum defects } & \multicolumn{2}{|c|}{ Energy Levels } & \multirow{2}{*}{$n$} & \multicolumn{2}{|c|}{ Quantum defects } & \multicolumn{2}{|c|}{ Energy Levels } \\
\hline & $\delta_{N I S T}$ & $\delta_{C a l}$ & $E_{N I S T}$ & $E_{C a l}$ & & $\delta_{N I S T}$ & $\delta_{C a l}$ & $E_{N I S T}$ & $E_{C a l}$ \\
\hline 4 & -0.00054 & -0.00054 & 102851.89 & 102851.89 & 33 & & -0.00125 & & 109607.85 \\
\hline 5 & -0.00067 & -0.00067 & 105320.31 & 105320.31 & 34 & & -0.00125 & & 109613.69 \\
\hline 6 & -0.00080 & -0.00080 & 106661.18 & 106661.18 & 35 & & -0.00125 & & 109619.04 \\
\hline 7 & & -0.00090 & & 107469.66 & 36 & & -0.00125 & & 109623.95 \\
\hline 8 & & -0.00098 & & 107994.39 & 37 & & -0.00125 & & 109628.46 \\
\hline 9 & & -0.00103 & & 108354.15 & 38 & & -0.00125 & & 109632.62 \\
\hline 10 & & -0.00108 & & 108611.48 & 39 & & -0.00126 & & 109636.47 \\
\hline 11 & & -0.00111 & & 108801.88 & 40 & & -0.00126 & & 109640.03 \\
\hline 12 & & -0.00113 & & 108946.70 & 41 & & -0.00126 & & 109643.34 \\
\hline 13 & & -0.00115 & & 109059.40 & 42 & & -0.00126 & & 109646.41 \\
\hline 14 & & -0.00117 & & 109148.83 & 43 & & -0.00126 & & 109649.27 \\
\hline 15 & & -0.00118 & & 109220.97 & 44 & & -0.00126 & & 109651.94 \\
\hline 16 & & -0.00119 & & 109280.02 & 45 & & -0.00126 & & 109654.43 \\
\hline 17 & & -0.00120 & & 109328.96 & 46 & & -0.00126 & & 109656.76 \\
\hline 18 & & -0.00121 & & 109369.97 & 47 & & -0.00126 & & 109658.94 \\
\hline 19 & & -0.00121 & & 109404.67 & 48 & & -0.00126 & & 109660.99 \\
\hline 20 & & -0.00122 & & 109434.31 & 49 & & -0.00126 & & 109662.91 \\
\hline 21 & & -0.00122 & & 109459.81 & 50 & & -0.00126 & & 109664.72 \\
\hline 22 & & -0.00123 & & 109481.91 & 51 & & -0.00126 & & 109666.43 \\
\hline 23 & & -0.00123 & & 109501.19 & 52 & & -0.00126 & & 109668.03 \\
\hline 24 & & -0.00123 & & 109518.12 & 53 & & -0.00126 & & 109669.55 \\
\hline 25 & & -0.00124 & & 109533.05 & 54 & & -0.00126 & & 109670.98 \\
\hline 26 & & -0.00124 & & 109546.30 & 55 & & -0.00126 & & 109672.34 \\
\hline 27 & & -0.00124 & & 109558.10 & 56 & & -0.00126 & & 109673.62 \\
\hline 28 & & -0.00124 & & 109568.66 & 57 & & -0.00126 & & 109674.84 \\
\hline 29 & & -0.00124 & & 109578.14 & 58 & & -0.00126 & & 109675.99 \\
\hline 30 & & -0.00125 & & 109586.69 & 59 & & -0.00126 & & 109677.09 \\
\hline 31 & & -0.00125 & & 109594.43 & 60 & & -0.00126 & & 109678.13 \\
\hline 32 & & -0.00125 & & 109601.46 & & & & & \\
\hline
\end{tabular}


Table VIII: Energy Level Sequence in $\mathrm{cm}^{-1}$ and Quantum Defects of $n f^{2} F_{7 / 2}^{o}(4 \leq n \leq 60)$ in Deuterium.

\begin{tabular}{|c|c|c|c|c|c|c|c|c|c|}
\hline \multirow{2}{*}{$n$} & \multicolumn{2}{|c|}{ Quantum defects } & \multicolumn{2}{|c|}{ Energy Levels } & \multirow{2}{*}{$n$} & \multicolumn{2}{|c|}{ Quantum defects } & \multicolumn{2}{|c|}{ Energy Levels } \\
\hline & $\delta_{N I S T}$ & $\delta_{C a l}$ & $E_{N I S T}$ & $E_{\text {Cal }}$ & & $\delta_{N I S T}$ & $\delta_{\text {Cal }}$ & $E_{N I S T}$ & $E_{C a l}$ \\
\hline 4 & -0.00054 & -0.00054 & 102851.89 & 102851.89 & 33 & & -0.00125 & & 109607.85 \\
\hline 5 & -0.00067 & -0.00067 & 105320.31 & 105320.31 & 34 & & -0.00125 & & 109613.69 \\
\hline 6 & -0.00081 & -0.00081 & 106661.18 & 106661.18 & 35 & & -0.00125 & & 109619.04 \\
\hline 7 & & -0.00091 & & 107469.66 & 36 & & -0.00126 & & 109623.95 \\
\hline 8 & & -0.00098 & & 107994.39 & 37 & & -0.00126 & & 109628.46 \\
\hline 9 & & -0.00104 & & 108354.15 & 38 & & -0.00126 & & 109632.62 \\
\hline 10 & & -0.00108 & & 108611.48 & 39 & & -0.00126 & & 109636.47 \\
\hline 11 & & -0.00111 & & 108801.88 & 40 & & -0.00126 & & 109640.03 \\
\hline 12 & & -0.00113 & & 108946.70 & 41 & & -0.00126 & & 109643.34 \\
\hline 13 & & -0.00115 & & 109059.40 & 42 & & -0.00126 & & 109646.41 \\
\hline 14 & & -0.00117 & & 109148.83 & 43 & & -0.00126 & & 109649.27 \\
\hline 15 & & -0.00118 & & 109220.97 & 44 & & -0.00126 & & 109651.94 \\
\hline 16 & & -0.00119 & & 109280.02 & 45 & & -0.00126 & & 109654.43 \\
\hline 17 & & -0.00120 & & 109328.96 & 46 & & -0.00126 & & 109656.76 \\
\hline 18 & & -0.00121 & & 109369.97 & 47 & & -0.00126 & & 109658.94 \\
\hline 19 & & -0.00121 & & 109404.67 & 48 & & -0.00126 & & 109660.99 \\
\hline 20 & & -0.00122 & & 109434.31 & 49 & & -0.00126 & & 109662.91 \\
\hline 21 & & -0.00122 & & 109459.81 & 50 & & -0.00126 & & 109664.72 \\
\hline 22 & & -0.00123 & & 109481.91 & 51 & & -0.00126 & & 109666.43 \\
\hline 23 & & -0.00123 & & 109501.19 & 52 & & -0.00126 & & 109668.03 \\
\hline 24 & & -0.00124 & & 109518.12 & 53 & & -0.00126 & & 109669.55 \\
\hline 25 & & -0.00124 & & 109533.05 & 54 & & -0.00126 & & 109670.98 \\
\hline 26 & & -0.00124 & & 109546.30 & 55 & & -0.00126 & & 109672.34 \\
\hline 27 & & -0.00124 & & 109558.10 & 56 & & -0.00126 & & 109673.62 \\
\hline 28 & & -0.00124 & & 109568.66 & 57 & & -0.00126 & & 109674.84 \\
\hline 29 & & -0.00125 & & 109578.14 & 58 & & -0.00126 & & 109675.99 \\
\hline 30 & & -0.00125 & & 109586.69 & 59 & & -0.00127 & & 109677.09 \\
\hline 31 & & -0.00125 & & 109594.43 & 60 & & -0.00127 & & 109678.13 \\
\hline 32 & & -0.00125 & & 109601.46 & & & & & \\
\hline
\end{tabular}

\subsection{Radiative Lifetimes with Natural line widths:}

Transition probabilities listed at NIST [23], the reference values of Radiative lifetimes for Deuterium are obtained. Utilizing these reference values of radiative lifetimes, the coefficients of expression 9 in text (Rykova's Expression) can be conveniently determined by least square fitting, are listed in Table IX.

\begin{tabular}{lcc} 
Table IX: Coefficients of Rykova's Expression. & \\
\hline \hline Energy Sequence & $\tau_{o(n S)}$ & $\alpha$ \\
\hline$n s^{2} S_{1 / 2}(n \geq 2)$ & $1.19564 \mathrm{E}-08$ & 2.1146 \\
$n P^{2} P^{o}(1 / 2,3 / 2)(n \geq 2)$ & $2.06236 \mathrm{E}-10$ & 2.9500 \\
$n d^{2} D_{(3 / 2,5 / 2)}(n \geq 3)$ & $6.08515 \mathrm{E}-10$ & 2.9449 \\
$n f^{2} F^{o}(5 / 2,7 / 2)(n \geq 4)$ & $1.21321 \mathrm{E}-09$ & 2.9510 \\
\hline \hline
\end{tabular}

Finally exploiting computed values of quantum defects listed in Tables: II-VIII and coefficients listed in Table IX. computed the radiative lifetimes up to $n=60$, with natural line widths for the following: $n s^{2} S_{1 / 2}(n \geq 2)$, $n p^{2} P_{(1 / 2,3 / 2)}^{o}(n \geq 2), n d^{2} D_{(3 / 2,5 / 2)}(n \geq 3)$, and $n f^{2} F_{(3 / 2,5 / 2)}^{o}(n \geq 4)$ sequence of deuterium Listed in Tables X-XIII. 
Table X: Radiative Lifetimes in $\mathrm{nS}$ and Natural linewidths in $\mathrm{cm}^{-1}$ of $n s^{2} S_{1 / 2}(2 \leq n \leq 60)$ in Deuterium.

\begin{tabular}{|c|c|c|c|c|c|c|c|}
\hline \multirow{2}{*}{$n$} & \multicolumn{2}{|c|}{ Radiative Lifetimes } & \multirow{2}{*}{$\frac{\text { Natural line Widths }}{\Gamma_{\text {Cal }}}$} & \multirow{2}{*}{$n$} & \multicolumn{2}{|c|}{ Radiative Lifetimes } & \multirow{2}{*}{$\begin{array}{c}\text { Natural line Widths } \\
\Gamma_{\text {Cal }}\end{array}$} \\
\hline & $\tau_{N I S T}$ & $\tau_{\mathrm{Cal}}$ & & & $\tau_{N I S T}$ & $\tau_{\text {Cal }}$ & \\
\hline 2 & & 51.793 & $2.036 \mathrm{E}-20$ & 32 & & 18214.614 & $5.790 \mathrm{E}-23$ \\
\hline 3 & & 122.078 & $8.639 \mathrm{E}-21$ & 33 & & 19439.206 & $5.425 \mathrm{E}-23$ \\
\hline 4 & 226.489 & 224.303 & 4.702E-21 & 34 & & 20705.868 & $5.093 \mathrm{E}-23$ \\
\hline 5 & 352.185 & 359.552 & $2.933 \mathrm{E}-21$ & 35 & & 22014.743 & $4.790 \mathrm{E}-23$ \\
\hline 6 & 534.972 & 528.681 & $1.995 \mathrm{E}-21$ & 36 & & 23365.972 & $4.513 \mathrm{E}-23$ \\
\hline 7 & & 732.406 & $1.440 \mathrm{E}-21$ & 37 & & 24759.693 & $4.259 \mathrm{E}-23$ \\
\hline 8 & & 971.345 & $1.086 \mathrm{E}-21$ & 38 & & 26196.038 & $4.026 \mathrm{E}-23$ \\
\hline 9 & & 1246.044 & $8.464 \mathrm{E}-22$ & 39 & & 27675.138 & $3.811 \mathrm{E}-23$ \\
\hline 10 & & 1556.988 & $6.773 \mathrm{E}-22$ & 40 & & 29197.122 & $3.612 \mathrm{E}-23$ \\
\hline 11 & & 1904.619 & $5.537 \mathrm{E}-22$ & 41 & & 30762.112 & $3.428 \mathrm{E}-23$ \\
\hline 12 & & 2289.341 & 4.607E-22 & 42 & & 32370.232 & $3.258 \mathrm{E}-23$ \\
\hline 13 & & 2711.525 & $3.889 \mathrm{E}-22$ & 43 & & 34021.600 & $3.100 \mathrm{E}-23$ \\
\hline 14 & & 3171.517 & $3.325 \mathrm{E}-22$ & 44 & & 35716.333 & $2.953 \mathrm{E}-23$ \\
\hline 15 & & 3669.640 & $2.874 \mathrm{E}-22$ & 45 & & 37454.546 & $2.816 \mathrm{E}-23$ \\
\hline 16 & & 4206.197 & $2.507 \mathrm{E}-22$ & 46 & & 39236.351 & $2.688 \mathrm{E}-23$ \\
\hline 17 & & 4781.473 & $2.206 \mathrm{E}-22$ & 47 & & 41061.857 & $2.568 \mathrm{E}-23$ \\
\hline 18 & & 5395.738 & $1.954 \mathrm{E}-22$ & 48 & & 42931.174 & $2.456 \mathrm{E}-23$ \\
\hline 19 & & 6049.249 & $1.743 \mathrm{E}-22$ & 49 & & 44844.405 & $2.352 \mathrm{E}-23$ \\
\hline 20 & & 6742.250 & $1.564 \mathrm{E}-22$ & 50 & & 46801.656 & $2.253 \mathrm{E}-23$ \\
\hline 21 & & 7474.973 & $1.411 \mathrm{E}-22$ & 51 & & 48803.028 & $2.161 \mathrm{E}-23$ \\
\hline 22 & & 8247.642 & $1.279 \mathrm{E}-22$ & 52 & & 50848.622 & $2.074 \mathrm{E}-23$ \\
\hline 23 & & 9060.469 & $1.164 \mathrm{E}-22$ & 53 & & 52938.536 & $1.992 \mathrm{E}-23$ \\
\hline 24 & & 9913.661 & $1.064 \mathrm{E}-22$ & 54 & & 55072.867 & $1.915 \mathrm{E}-23$ \\
\hline 25 & & 10807.415 & $9.758 \mathrm{E}-23$ & 55 & & 57251.710 & $1.842 \mathrm{E}-23$ \\
\hline 26 & & 11741.920 & $8.981 \mathrm{E}-23$ & 56 & & 59475.159 & $1.773 \mathrm{E}-23$ \\
\hline 27 & & 12717.361 & $8.293 \mathrm{E}-23$ & 57 & & 61743.306 & $1.708 \mathrm{E}-23$ \\
\hline 28 & & 13733.914 & 7.679E-23 & 58 & & 64056.242 & $1.646 \mathrm{E}-23$ \\
\hline 29 & & 14791.752 & $7.130 \mathrm{E}-23$ & 59 & & 66414.057 & $1.588 \mathrm{E}-23$ \\
\hline 30 & & 15891.042 & $6.636 \mathrm{E}-23$ & 60 & & 68816.837 & $1.532 \mathrm{E}-23$ \\
\hline 31 & & 17031.943 & $6.192 \mathrm{E}-23$ & & & & \\
\hline
\end{tabular}


Table XI: Radiative Lifetimes in $\mathrm{nS}$ and Natural linewidths in $\mathrm{cm}^{-1}$ of $n p^{2} P_{(1 / 2,3 / 2)}^{o}(2 \leq n \leq 60)$ in Deuterium.

\begin{tabular}{|c|c|c|c|c|c|c|c|}
\hline \multirow{2}{*}{$n$} & \multicolumn{2}{|c|}{ Radiative Lifetimes } & \multirow{2}{*}{$\begin{array}{c}\text { Natural line Widths } \\
\Gamma_{\text {Cal }}\end{array}$} & \multirow{2}{*}{$n$} & \multicolumn{2}{|c|}{ Radiative Lifetimes } & \multirow{2}{*}{$\frac{\text { Natural line Widths }}{\Gamma_{\mathrm{Cal}}}$} \\
\hline & $\tau_{N I S T}$ & $\tau_{C a l}$ & & & $\tau_{N I S T}$ & $\tau_{C a l}$ & \\
\hline 2 & 1.596 & 1.594 & $6.615 \mathrm{E}-19$ & 32 & & 5683.271 & $1.856 \mathrm{E}-22$ \\
\hline 3 & 5.270 & 5.273 & $2.000 \mathrm{E}-19$ & 33 & & 6223.301 & $1.695 \mathrm{E}-22$ \\
\hline 4 & 12.302 & 12.320 & $8.560 \mathrm{E}-20$ & 34 & & 6796.203 & $1.552 \mathrm{E}-22$ \\
\hline 5 & 23.783 & 23.795 & 4.432E-20 & 35 & & 7402.922 & $1.425 \mathrm{E}-22$ \\
\hline 6 & 40.808 & 40.745 & $2.588 \mathrm{E}-20$ & 36 & & 8044.403 & $1.311 \mathrm{E}-22$ \\
\hline 7 & & 64.203 & $1.643 \mathrm{E}-20$ & 37 & & 8721.587 & $1.209 \mathrm{E}-22$ \\
\hline 8 & & 95.196 & $1.108 \mathrm{E}-20$ & 38 & & 9435.418 & $1.118 \mathrm{E}-22$ \\
\hline 9 & & 134.744 & $7.827 \mathrm{E}-21$ & 39 & & 10186.834 & $1.035 \mathrm{E}-22$ \\
\hline 10 & & 183.858 & $5.736 \mathrm{E}-21$ & 40 & & 10976.775 & $9.607 \mathrm{E}-23$ \\
\hline 11 & & 243.547 & $4.330 \mathrm{E}-21$ & 41 & & 11806.179 & 8.933E-23 \\
\hline 12 & & 314.812 & $3.350 \mathrm{E}-21$ & 42 & & 12675.983 & $8.320 \mathrm{E}-23$ \\
\hline 13 & & 398.652 & $2.645 \mathrm{E}-21$ & 43 & & 13587.121 & $7.762 \mathrm{E}-23$ \\
\hline 14 & & 496.058 & $2.126 \mathrm{E}-21$ & 44 & & 14540.529 & $7.253 \mathrm{E}-23$ \\
\hline 15 & & 608.021 & $1.734 \mathrm{E}-21$ & 45 & & 15537.139 & $6.788 \mathrm{E}-23$ \\
\hline 16 & & 735.527 & $1.434 \mathrm{E}-21$ & 46 & & 16577.884 & $6.361 \mathrm{E}-23$ \\
\hline 17 & & 879.558 & $1.199 \mathrm{E}-21$ & 47 & & 17663.695 & $5.970 \mathrm{E}-23$ \\
\hline 18 & & 1041.095 & $1.013 \mathrm{E}-21$ & 48 & & 18795.503 & $5.611 \mathrm{E}-23$ \\
\hline 19 & & 1221.113 & $8.636 \mathrm{E}-22$ & 49 & & 19974.236 & $5.280 \mathrm{E}-23$ \\
\hline 20 & & 1420.588 & 7.424E-22 & 50 & & 21200.823 & $4.974 \mathrm{E}-23$ \\
\hline 21 & & 1640.490 & $6.429 \mathrm{E}-22$ & 51 & & 22476.190 & 4.692E-23 \\
\hline 22 & & 1881.789 & $5.604 \mathrm{E}-22$ & 52 & & 23801.265 & $4.431 \mathrm{E}-23$ \\
\hline 23 & & 2145.451 & $4.915 \mathrm{E}-22$ & 53 & & 25176.973 & $4.189 \mathrm{E}-23$ \\
\hline 24 & & 2432.442 & $4.336 \mathrm{E}-22$ & 54 & & 26604.238 & $3.964 \mathrm{E}-23$ \\
\hline 25 & & 2743.722 & $3.844 \mathrm{E}-22$ & 55 & & 28083.984 & $3.755 \mathrm{E}-23$ \\
\hline 26 & & 3080.254 & $3.424 \mathrm{E}-22$ & 56 & & 29617.135 & $3.561 \mathrm{E}-23$ \\
\hline 27 & & 3442.996 & $3.063 \mathrm{E}-22$ & 57 & & 31204.611 & $3.380 \mathrm{E}-23$ \\
\hline 28 & & 3832.903 & $2.751 \mathrm{E}-22$ & 58 & & 32847.334 & $3.211 \mathrm{E}-23$ \\
\hline 29 & & 4250.932 & $2.481 \mathrm{E}-22$ & 59 & & 34546.224 & $3.053 \mathrm{E}-23$ \\
\hline 30 & & 4698.036 & $2.245 \mathrm{E}-22$ & 60 & & 36302.202 & $2.905 \mathrm{E}-23$ \\
\hline 31 & & 5175.165 & $2.038 \mathrm{E}-22$ & & & & \\
\hline
\end{tabular}


Table XII: Radiative Lifetimes in $\mathrm{nS}$ and Natural linewidths $\mathrm{cm}^{-1}$ of $n d^{2} D_{(3 / 2,5 / 2)}(3 \leq n \leq 60)$ in Deuterium.

\begin{tabular}{|c|c|c|c|c|c|c|}
\hline \multirow{2}{*}{$n$} & \multicolumn{2}{|c|}{ Radiative Lifetimes } & \multirow{2}{*}{$\frac{\text { Natural line Widths }}{\Gamma_{\mathrm{Cal}}}$} & \multirow{2}{*}{$n$} & Radiative Lifetimes & \multirow{2}{*}{$\frac{\text { Natural line Widths }}{\Gamma_{\text {Cal }}}$} \\
\hline & $\tau_{N I S T}$ & $\tau_{C a l}$ & & & $\tau_{N I S T}$ & \\
\hline 3 & 15.463 & 15.471 & $6.817 \mathrm{E}-20$ & 32 & 16475.555 & $6.401 \mathrm{E}-23$ \\
\hline 4 & 36.139 & 36.095 & $2.922 \mathrm{E}-20$ & 33 & 18038.235 & $5.846 \mathrm{E}-23$ \\
\hline 5 & 69.651 & 69.637 & $1.514 \mathrm{E}-20$ & 34 & 19695.779 & $5.354 \mathrm{E}-23$ \\
\hline 6 & 119.084 & 119.129 & $8.852 \mathrm{E}-21$ & 35 & 21450.899 & $4.916 \mathrm{E}-23$ \\
\hline 7 & & 187.571 & $5.622 \mathrm{E}-21$ & 36 & 23306.306 & $4.525 \mathrm{E}-23$ \\
\hline 8 & & 277.932 & $3.794 \mathrm{E}-21$ & 37 & 25264.705 & 4.174E-23 \\
\hline 9 & & 393.160 & $2.682 \mathrm{E}-21$ & 38 & 27328.797 & $3.859 \mathrm{E}-23$ \\
\hline 10 & & 536.182 & $1.967 \mathrm{E}-21$ & 39 & 29501.279 & $3.575 \mathrm{E}-23$ \\
\hline 11 & & 709.907 & $1.486 \mathrm{E}-21$ & 40 & 31784.845 & $3.318 \mathrm{E}-23$ \\
\hline 12 & & 917.227 & $1.150 \mathrm{E}-21$ & 41 & 34182.183 & $3.085 \mathrm{E}-23$ \\
\hline 13 & & 1161.022 & $9.083 \mathrm{E}-22$ & 42 & 36695.979 & $2.874 \mathrm{E}-23$ \\
\hline 14 & & 1444.158 & $7.302 \mathrm{E}-22$ & 43 & 39328.916 & $2.681 \mathrm{E}-23$ \\
\hline 15 & & 1769.487 & $5.960 \mathrm{E}-22$ & 44 & 42083.672 & $2.506 \mathrm{E}-23$ \\
\hline 16 & & 2139.851 & $4.928 \mathrm{E}-22$ & 45 & 44962.923 & $2.345 \mathrm{E}-23$ \\
\hline 17 & & 2558.083 & $4.123 \mathrm{E}-22$ & 46 & 47969.339 & $2.198 \mathrm{E}-23$ \\
\hline 18 & & 3027.004 & $3.484 \mathrm{E}-22$ & 47 & 51105.589 & $2.064 \mathrm{E}-23$ \\
\hline 19 & & 3549.428 & $2.971 \mathrm{E}-22$ & 48 & 54374.339 & $1.940 \mathrm{E}-23$ \\
\hline 20 & & 4128.157 & $2.555 \mathrm{E}-22$ & 49 & 57778.252 & $1.825 \mathrm{E}-23$ \\
\hline 21 & & 4765.989 & $2.213 \mathrm{E}-22$ & 50 & 61319.985 & $1.720 \mathrm{E}-23$ \\
\hline 22 & & 5465.712 & $1.929 \mathrm{E}-22$ & 51 & 65002.196 & $1.622 \mathrm{E}-23$ \\
\hline 23 & & 6230.107 & $1.693 \mathrm{E}-22$ & 52 & 68827.537 & $1.532 \mathrm{E}-23$ \\
\hline 24 & & 7061.948 & $1.493 \mathrm{E}-22$ & 53 & 72798.659 & $1.449 \mathrm{E}-23$ \\
\hline 25 & & 7964.002 & $1.324 \mathrm{E}-22$ & 54 & 76918.209 & $1.371 \mathrm{E}-23$ \\
\hline 26 & & 8939.031 & $1.180 \mathrm{E}-22$ & 55 & 81188.832 & $1.299 \mathrm{E}-23$ \\
\hline 27 & & 9989.789 & $1.056 \mathrm{E}-22$ & 56 & 85613.171 & $1.232 \mathrm{E}-23$ \\
\hline 28 & & 11119.026 & $9.485 \mathrm{E}-23$ & 57 & 90193.864 & $1.169 \mathrm{E}-23$ \\
\hline 29 & & 12329.486 & $8.553 \mathrm{E}-23$ & 58 & 94933.550 & $1.111 \mathrm{E}-23$ \\
\hline 30 & & 13623.906 & $7.741 \mathrm{E}-23$ & 59 & 99834.861 & $1.056 \mathrm{E}-23$ \\
\hline 31 & & 15005.020 & $7.028 \mathrm{E}-23$ & 60 & 104900.429 & $1.005 \mathrm{E}-23$ \\
\hline
\end{tabular}


Table XIII: Radiative Lifetimes in $\mathrm{nS}$ and Natural linewidths $\mathrm{cm}^{-1} n f^{2} F_{(5 / 2,7 / 2)}^{o}(4 \leq n \leq 60)$ in Deuterium.

\begin{tabular}{|c|c|c|c|c|c|c|}
\hline \multirow{2}{*}{$n$} & \multicolumn{2}{|c|}{ Radiative Lifetimes } & \multirow{2}{*}{$\frac{\text { Natural line Widths }}{\Gamma_{\mathrm{Cal}}}$} & \multirow{2}{*}{$n$} & Radiative Lifetimes & \multirow{2}{*}{$\frac{\text { Natural line Widths }}{\Gamma_{\text {Cal }}}$} \\
\hline & $\tau_{N I S T}$ & $\tau_{\mathrm{Cal}}$ & & & $\tau_{N I S T}$ & \\
\hline 4 & 72.509 & 72.575 & $1.453 \mathrm{E}-20$ & 33 & 36738.165 & $2.871 \mathrm{E}-23$ \\
\hline 5 & 140.281 & 140.206 & $7.522 \mathrm{E}-21$ & 34 & 40121.367 & $2.629 \mathrm{E}-23$ \\
\hline 6 & 239.880 & 240.121 & 4.392E-21 & 35 & 43704.376 & $2.413 \mathrm{E}-23$ \\
\hline 7 & & 378.429 & $2.787 \mathrm{E}-21$ & 36 & 47492.777 & $2.221 \mathrm{E}-23$ \\
\hline 8 & & 561.190 & $1.879 \mathrm{E}-21$ & 37 & 51492.147 & $2.048 \mathrm{E}-23$ \\
\hline 9 & & 794.422 & $1.327 \mathrm{E}-21$ & 38 & 55708.054 & $1.893 \mathrm{E}-23$ \\
\hline 10 & & 1084.107 & $9.728 \mathrm{E}-22$ & 39 & 60146.061 & $1.753 \mathrm{E}-23$ \\
\hline 11 & & 1436.194 & 7.343E-22 & 40 & 64811.722 & $1.627 \mathrm{E}-23$ \\
\hline 12 & & 1856.603 & $5.680 \mathrm{E}-22$ & 41 & 69710.584 & $1.513 \mathrm{E}-23$ \\
\hline 13 & & 2351.227 & $4.485 \mathrm{E}-22$ & 42 & 74848.190 & $1.409 \mathrm{E}-23$ \\
\hline 14 & & 2925.937 & $3.604 \mathrm{E}-22$ & 43 & 80230.072 & $1.314 \mathrm{E}-23$ \\
\hline 15 & & 3586.579 & $2.940 \mathrm{E}-22$ & 44 & 85861.759 & $1.228 \mathrm{E}-23$ \\
\hline 16 & & 4338.980 & $2.431 \mathrm{E}-22$ & 45 & 91748.772 & $1.149 \mathrm{E}-23$ \\
\hline 17 & & 5188.949 & $2.032 \mathrm{E}-22$ & 46 & 97896.626 & $1.077 \mathrm{E}-23$ \\
\hline 18 & & 6142.276 & $1.717 \mathrm{E}-22$ & 47 & 104310.831 & $1.011 \mathrm{E}-23$ \\
\hline 19 & & 7204.734 & $1.464 \mathrm{E}-22$ & 48 & 110996.891 & $9.501 \mathrm{E}-24$ \\
\hline 20 & & 8382.081 & $1.258 \mathrm{E}-22$ & 49 & 117960.303 & $8.940 \mathrm{E}-24$ \\
\hline 21 & & 9680.059 & $1.089 \mathrm{E}-22$ & 50 & 125206.559 & $8.423 \mathrm{E}-24$ \\
\hline 22 & & 11104.399 & $9.497 \mathrm{E}-23$ & 51 & 132741.145 & $7.945 \mathrm{E}-24$ \\
\hline 23 & & 12660.815 & $8.330 \mathrm{E}-23$ & 52 & 140569.544 & $7.502 \mathrm{E}-24$ \\
\hline 24 & & 14355.010 & 7.347E-23 & 53 & 148697.231 & $7.092 \mathrm{E}-24$ \\
\hline 25 & & 16192.675 & $6.513 \mathrm{E}-23$ & 54 & 157129.676 & $6.712 \mathrm{E}-24$ \\
\hline 26 & & 18179.489 & $5.801 \mathrm{E}-23$ & 55 & 165872.347 & $6.358 \mathrm{E}-24$ \\
\hline 27 & & 20321.120 & $5.190 \mathrm{E}-23$ & 56 & 174930.702 & $6.029 \mathrm{E}-24$ \\
\hline 28 & & 22623.225 & $4.662 \mathrm{E}-23$ & 57 & 184310.200 & $5.722 \mathrm{E}-24$ \\
\hline 29 & & 25091.450 & $4.203 \mathrm{E}-23$ & 58 & 194016.290 & $5.436 \mathrm{E}-24$ \\
\hline 30 & & 27731.434 & $3.803 \mathrm{E}-23$ & 59 & 204054.419 & $5.168 \mathrm{E}-24$ \\
\hline 31 & & 30548.804 & $3.452 \mathrm{E}-23$ & 60 & 214430.029 & $4.918 \mathrm{E}-24$ \\
\hline 32 & & 33549.178 & $3.143 \mathrm{E}-23$ & & & \\
\hline
\end{tabular}

All ' $n l$ ' sequence radiative lifetimes of Deuterium show a good agreement with refence values [23] and follows the simple scaling law $(n-\delta)^{\alpha}$, with their uncertainty in energies due to uncertainty principle. The uncertainties that is natural line width shows that all transitions have negligible natural broadening. In result the spectrum of Deuterium must have sharp spectral lines. The radiative lifetimes and Natural line widths for high ' $n$ ' values are presented very first time.

\subsection{Graphical Representation:}

Graph I: Quantum defects as a function of Principal quantum numbers ( $n-\delta$ curves).

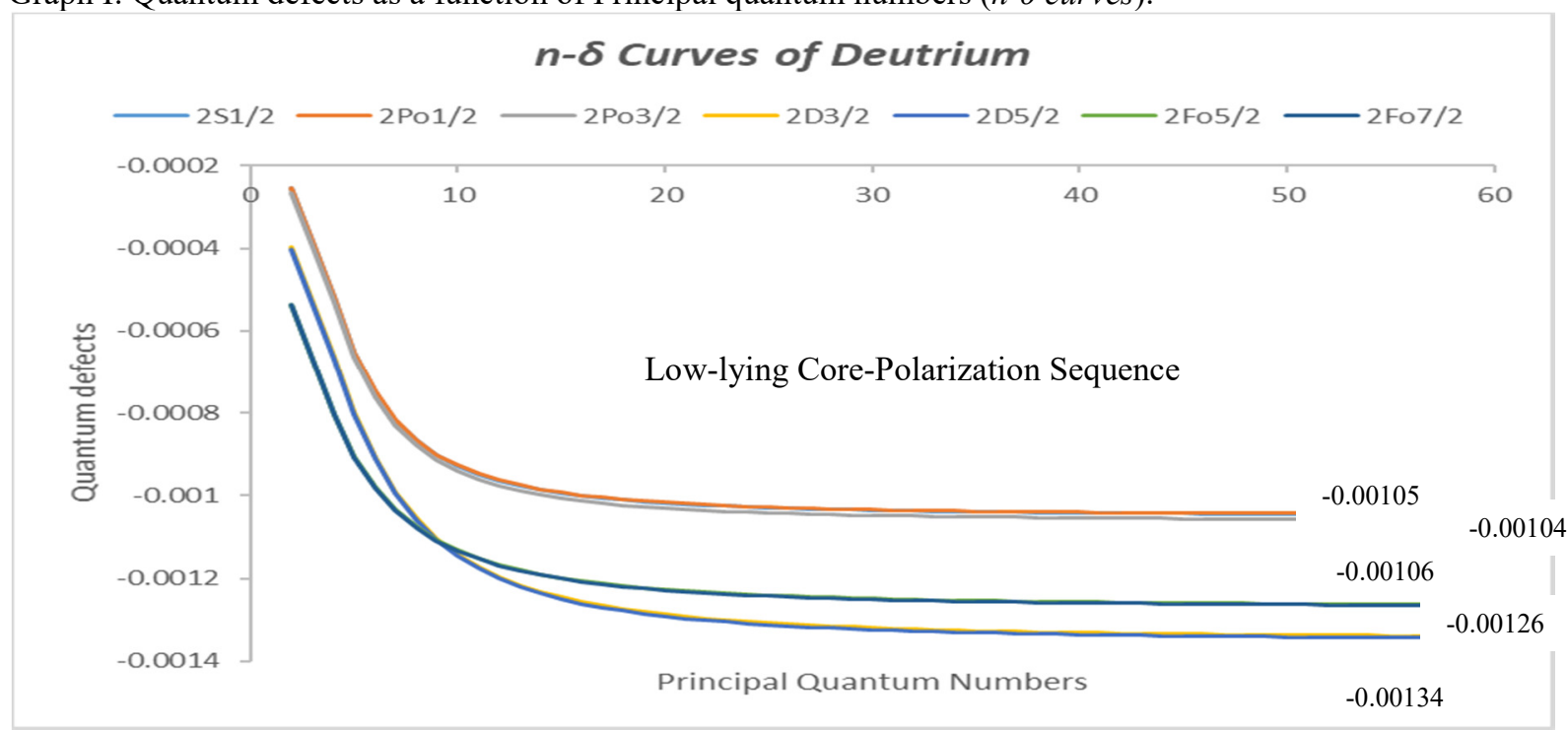


From the comparison of coefficient of equation 6 in text ' $a_{0}$ ' with the quantum defects of lowest possible state of each series that is ' $\delta_{0}$ ' and highest state up to which series is computed that is ' $\delta_{60}$ '. We can clearly see that quantum defects of all ' $n l$ ' sequence of Deuterium converges towards the coefficient ' $a_{0}$ '. can also be clearly seen in Graph I. The core-polarization nature of all ' $n l$ ' sequence of Deuterium clearly displays in Graph I. Further analysation of the $n-\delta$ curves in Graph I, reveals that in all sequence, quantum defects ' $\delta_{n}$ ', first exponentially decrease and then becomes constant and shows asymptotic behaviour due to the continuous change in quantum defects.

From above $n-\delta$ curves we can also see that $n s^{2} S_{1 / 2}$ sharply overlapped $n p^{2} P_{1 / 2}^{o}$, and in these $n d^{2} D_{(3 / 2,5 / 2) \text {, }}$ $n f^{2} F^{o}{ }_{(5 / 2,7 / 2)}$ sequences $J=l \pm 1 / 2$ sharply overlaps each other and there is a fine splitting between ${ }^{2} P^{o} 1 / 2$, and ${ }^{2} P^{o}{ }_{3 / 2}$. The quantum defects sequence of $n f^{2} F_{(5 / 2,7 / 2)}^{o}$ lies above $n d^{2} D_{(3 / 2,5 / 2)}$.

Graph II: Radiative Lifetimes as a function of principal quantum numbers ( $n$ - $\tau$ curves).

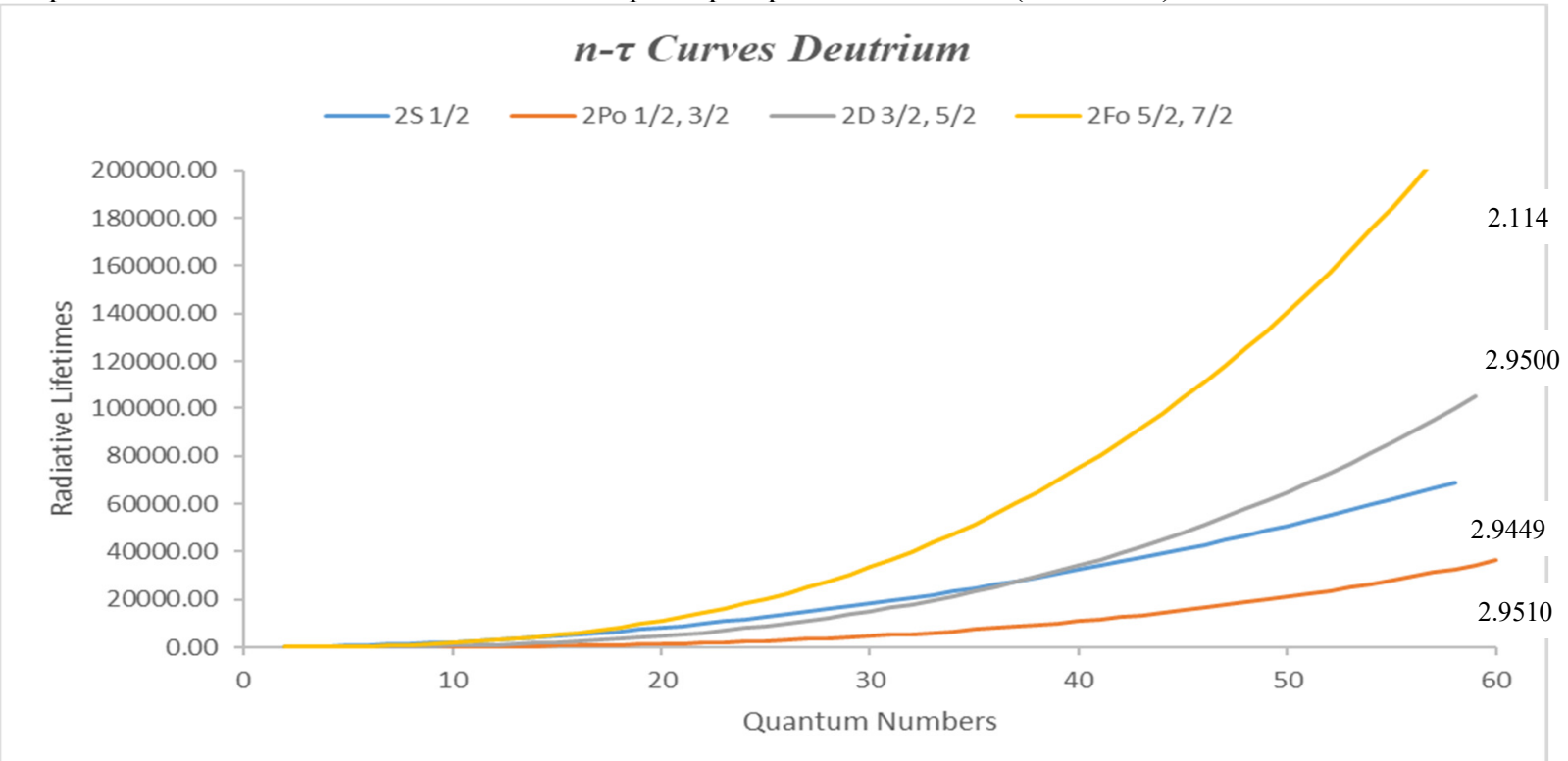

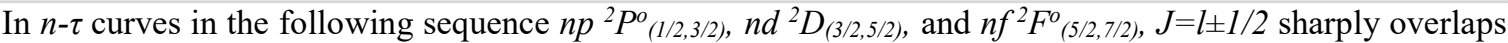
each other and shows exponentially increasing behaviour. Among all sequence $n f^{2} F^{o}(5 / 2,7 / 2)$ has the steepest curve but series: $n s^{2} S_{1 / 2}$ and, $n d^{2} D_{(3 / 2,7 / 2)}$ are closest and $n p^{2} P_{(1 / 2,3 / 2)}^{o}$ has the lowest curve. The Graph II. also shows that $s, p, d$ and $f$ sequence follow the simple scaling law $\left(n-\delta_{n}\right)^{\alpha}$. The values of ' $\alpha$ ' for ' $n l$ ' sequence of radiative lifetimes are explicitly shows in Graph II. 
Graph III: Grotrian Energy Level Diagram for $s, p, d$ and $f$ Sequence of Deuterium.
Energy in $\mathrm{cm}^{-1}$
$n s^{2} S_{L / 2}$
$n p^{2} P^{o}(1 / 2,3 / 2)$
$n d^{2} D_{(3 / 2,5 / 2)}$
$n f^{2} F_{(5 / 2,7 / 2)}$

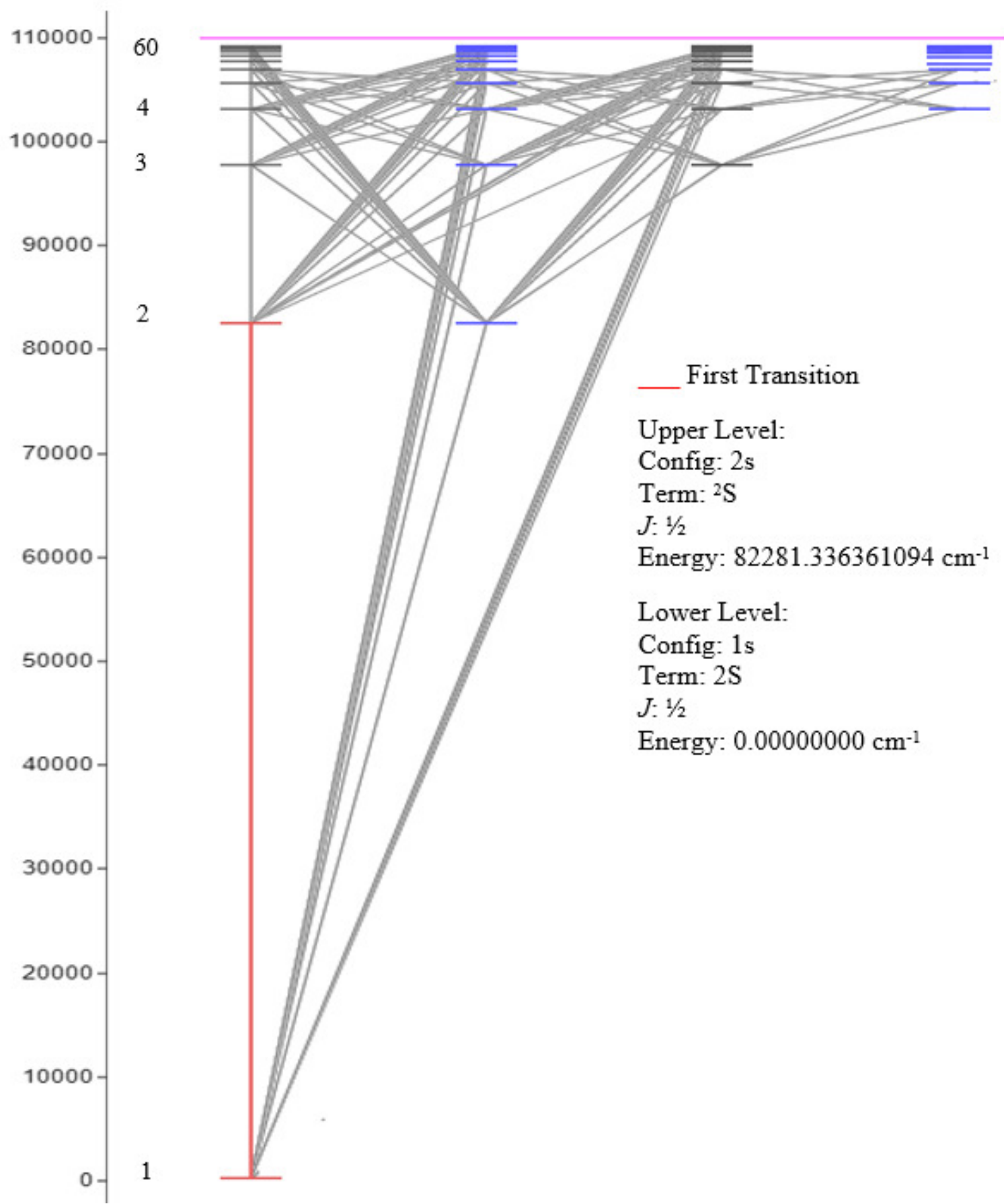

A summarising view of all ' $n l$ ' energy levels sequences of Deuterium are shown in Grotrian energy level diagram. From Graph III we can deduce that $s, p, d$ and $f$ energy levels sequence of Deuterium are same as hydrogen with bound and $l$ degenerated states for higher ' $n$ ' levels and all the transitions obeys the selection rule $J=0, \pm 1$, \pm 2 . It also shows that for all ' $n l$ ' energy levels sequence of Deuterium the quantum defect is approximately equal to zero.

\section{Conclusion}

The energy level sequence with Quantum defects and Radiative lifetimes with Natural line widths for the following sequence: $n s^{2} S_{1 / 2}, n p^{2} P_{(1 / 2,3 / 2), n d^{2} D_{(3 / 2,5 / 2)} \text { and, } n f^{2} F^{o}(5 / 2,7 / 2)}$ up to $n=60$, in deuterium are presented. Both sets of that compared with their experimental values obtained from NIST[23]. Quite an excellent agreement found between experimental and computed values in this work. 
Conclusive remarks about theoretical computation of deuterium are as:

i. The deviation of this work is less than $0.1 \mathrm{~cm}^{-1}$ for energy levels sequence of Deuterium and 0.001 for quantum defects.

ii. Transition probabilities $A_{i k}$ listed at NIST [23] were utilized to obtained first few experimental values of radiative Lifetimes of all ' $n l$ ' sequence in deuterium. Then by least square fitting of data by using equation 9 in text, radiative lifetimes were computed for up to $n=60$ quantum number. The deviation of radiative lifetimes in this work is less than $0.1 \mathrm{nS}$ in $p, d$ and $f$ sequence except for $s$ sequence.

iii. From Table I and Graph I in text we can clearly infer that al ' $n l$ ' sequence of Deuterium are converges towards the Martin's expression coefficient ' $a_{0}$ ' and the negative sign of ' $a_{0}$ ' indicates that all ' $n l$ ' sequence are low lying core polarization sequence.

iv. In all ' $n l^{\prime}$ ' sequence of quantum defects of: $n s^{2} S_{1 / 2}$ sharply overlaps $n p^{2} P^{o}{ }_{1 / 2}$, and in $n d^{2} D_{3 / 2,5 / 2}, n f^{2} F^{o}{ }_{5 / 2,7 / 2}$ sequences quantum defects of $J=l \pm 1 / 2$ sharply overlaps each other and there is a fine splitting between $n p^{2} P_{1 / 2}^{o}$ and $n p^{2} P^{o}{ }_{3 / 2}$ (see Graph. I)

v. In all ' $n l$ ' sequence of radiative lifetimes $J=l \pm 1 / 2$ sharply overlaps each other except $n s$ sequence and shows exponentially increasing behaviour. Among them the $n f^{2} F^{o}{ }_{5 / 2,7 / 2}$ has the steepest curve but series: $n s^{2} S_{1 / 2}$ and, $n d^{2} D_{3 / 2,5 / 2}$ are closest (see Graph. III).

vi. All ' $n l$ ' sequences of radiative lifetimes in Deuterium follows the simple scaling law $\left(n-\delta_{n}\right)^{\alpha}$.

vii. The Natural line widths computed based on principal of uncertainty show negligible values. Means natural broadening is approximately zero which shows the Deuterium spectrum is a sharp line spectrum (see Graph. III).

viii. The Grotrian diagram clearly displays the approximately zero quantum defects of deuterium ' $n l$ ' sequence and the degeneracy of states for higher $\mathrm{n}$ values (see Graph. II).

ix. Finally, In Grotrian diagram for all the allowed transitions data available at NIST [23] are shown, following the selection rule $J=0, \pm 1, \pm 2$.

x. This theoretical computation is also a continuation of the work by Raza. S. et al. in Neutral Hydrogen [3].

\section{Appendix A. Supplementary Data:}

Related to this work, all the supplementary Data can be easily available online at: https://www.physics.nist.gov/PhysRefData/ASD/lines form.html

\section{Explanation of Tables:}

Table I. Shows the Coefficients of Martin's Expression \& Sequence Convergence ( Limit:109708.61455299 $\mathrm{cm}^{-1}$ )

Energy Sequence: Energy levels sequence of Deuterium with initial principal quantum number $a_{0}$ : Coefficient of Martin's expression for ' $n l^{\prime}$ ' sequence of Deuterium.

$a_{1}$ : Coefficient of Martin's expression for ' $n l$ ' sequence of Deuterium.

$a_{2}$ : Coefficient of Martin's expression for ' $n l$ ' sequence of Deuterium.

$a_{3}$ : Coefficient of Martin's expression for ' $n l^{\prime}$ 'sequence of Deuterium.

$\delta_{0}$ : Quantum defects of lowest possible state of each series of Deuterium.

$\delta_{60}:$ Quantum defects of highest possible state up to which sequence is computed.

Convergence Nature: Shows the dominating potential in which electron revolves.

Table II Energy Level sequence and Quantum Defects of $n s^{2} S_{1 / 2}(2 \leq n \leq 60)$ in Deuterium.

n: Principal Quantum number.

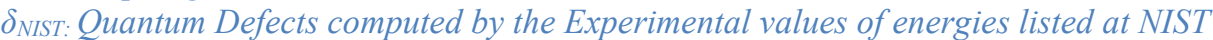

$\delta_{\text {Cal: }}$ Quantum Defects calculated in this work by WBEPMT

$E_{N I S T:}$ Energy levels Listed at NIST

$E_{\text {cal: }}$ Energy levels calculated in this work by WBEPMT

Table III Energy Level sequence and Quantum Defects of $n s^{2} P^{o}{ }_{1 / 2}(2 \leq n \leq 60)$ in Deuterium.

n: Principal Quantum number.

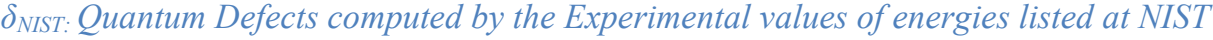

$\delta_{\text {Cal: }}$ Quantum Defects calculated in this work by WBEPMT

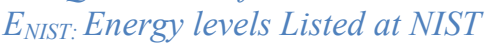

$E_{\text {cal: }}$ Energy levels calculated in this work by WBEPMT

Table IV Energy Level sequence and Quantum Defects of $n s^{2} P^{o}{ }_{3 / 2}(2 \leq n \leq 60)$ in Deuterium

n: Principal Quantum number.

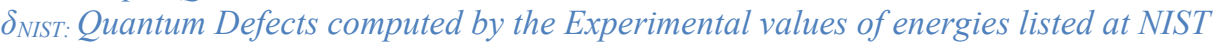

$\delta_{\text {Cal: }}$ Quantum Defects calculated in this work by WBEPMT

$E_{N I S T:}$ Energy levels Listed at NIST 
$E_{\text {cal: }}$ Energy levels calculated in this work by WBEPMT

Table V Energy Level Sequence and Quantum Defects of $n s^{2} D_{3 / 2}(3 \leq n \leq 60)$ in Deuterium. n: Principal Quantum number.

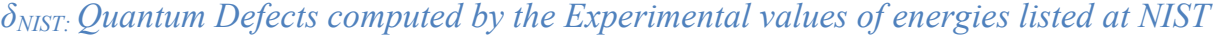

$\delta_{\text {Cal: }}$ Quantum Defects calculated in this work by WBEPMT

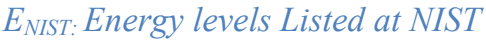

$E_{\text {cal: }}$ Energy levels calculated in this work by WBEPMT

Table VI Energy Level Sequence and Quantum Defects of $n s^{2} D_{5 / 2}(3 \leq n \leq 60)$ in Deuterium.

n: Principal Quantum number.

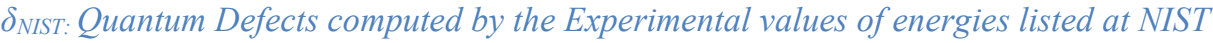

$\delta_{\text {Cal: }}$ Quantum Defects calculated in this work by WBEPMT

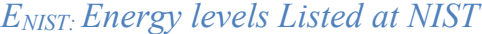

$E_{\text {cal: }}$ Energy levels calculated in this work by WBEPMT

Table VII Energy Level Sequence and Quantum Defects of $n s^{2} F^{0}{ }_{5 / 2}(4 \leq n \leq 60)$ in Deuterium. n: Principal Quantum number.

$\delta_{\text {NIST: }}$ Quantum Defects computed by the Experimental values of energies listed at NIST

$\delta_{\text {Cal: }}$ Quantum Defects calculated in this work by WBEPMT

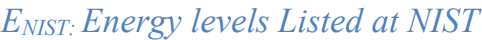

$E_{\text {cal: }}$ Energy levels calculated in this work by WBEPMT

Table

VIII

\section{Energy Level Sequence and Quantum Defects of $n s^{2} D_{7 / 2}(4 \leq n \leq 60)$ in Deuterium}

n: Principal Quantum number.

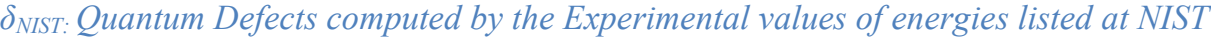

$\delta_{\text {Cal: }}$ Quantum Defects calculated in this work by WBEPMT

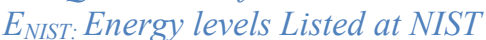

$E_{\text {cal: }}$ Energy levels calculated in this work by WBEPMT

Table IX. Coefficients of Rykova's Expression.

Energy Sequence: Energy levels sequence of Deuterium with initial principal quantum number $\tau_{0}$ : Coefficients of Rykova's Expression for measuring radiative lifetimes.

$\alpha$ : Power of effective quantum number $n$ *, for which Radiative lifetimes are directly proportional.

Table X. Radiative Lifetimes in $\mathrm{nS}$ and Natural linewidths in $\mathrm{cm}^{-1}$ of ${ }^{2} S_{1 / 2}(2 \leq n \leq 60)$ in Deuterium.

n: Principal Quantum number.

$\tau_{\text {NIIST }}$ : Experimental values of radiative lifetimes obtained from Transition probabilities listed at NIST.

$\tau_{\text {cal: }}$ Radiative lifetimes computed by exploiting Rykova's expression and WBEPMT.

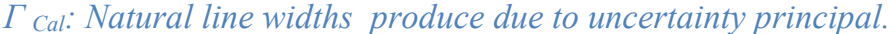

Table XI. Radiative Lifetimes in $\mathrm{nS}$ and Natural linewidths in $\mathrm{cm}^{-1}$ of ${ }^{2} P_{(1 / 2,3 / 2)}(2 \leq n \leq 60)$ in Deuterium. n: Principal Quantum number

$\tau_{\text {NIIST }}:$ Experimental values of radiative lifetimes obtained from Transition probabilities listed at NIST.

$\tau_{\text {cal: }}$ Radiative lifetimes computed by exploiting Rykova's expression and WBEPMT.

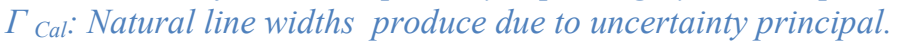

Table

XII.

Radiative Lifetimes in $\mathrm{nS}$ and Natural linewidths $\mathrm{cm}^{-1}$ of ${ }^{2} D_{(3 / 2,5 / 2)}(2 \leq n \leq 60)$ in Deuterium.

n: Principal Quantum number.

$\tau_{N I I S T}$ : Experimental values of radiative lifetimes obtained from Transition probabilities listed at NIST.

$\tau_{\text {cal: }}$ Radiative lifetimes computed by exploiting Rykova's expression and WBEPMT.

$\Gamma$ Cal: Natural line widths produce due to uncertainty principal.

Table

Radiative Lifetimes in $\mathrm{nS}$ and Natural linewidths $\mathrm{cm}^{-1}$ of ${ }^{2} F^{0}(5 / 2,7 / 2)(2 \leq n \leq 60)$ in Deuterium.

n: Principal Quantum number.

$\tau_{\text {NIIST }}$ : Experimental values of radiative lifetimes obtained from Transition probabilities listed at NIST.

$\tau_{\text {cal: }}$ Radiative lifetimes computed by exploiting Rykova's expression and WBEPMT.

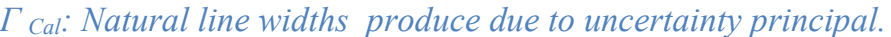

7. References:

[1] https://en.wikipedia.org/wiki/Deuterium\#Applications. 
[2] Zheng NW, Wang T, Ma DX, Zhou T, Fan J. Weakest bound electron potential model theory. International journal of quantum chemistry. 2004;98(3):281-90.

[3] Raza. S, Ali. N, Hameed. M N. Spectral Energies and Radiative Lifetimes of Rydberg States in Neutral Hydrogen. The Journal of Natural Sciences Research. 2018 Aug, Volume 8,(No 14): 37-45.

[4] Ali. N, Hameed. M N, Raza. S, Theoretical Investigation of Radiative Lifetimes and Rydberg Levels Sequence in Indium I. The Journal of Natural Sciences Research. 2018 Oct, Volume 8,(No 17): 29-50.

[5] Ateş Ş, Uğurtan HH. Lifetimes of excited levels for atomic silicon. Indian Journal of Physics. 2013 Jan 1;87(1):9-17.

[6] Çelik G, Doğan D, Ateş Ş, Taşer M. Transition probabilities and radiative lifetimes of levels in FI. Atomic Data and Nuclear Data Tables. 2012 Jul 1;98(4):566-88.

[7] Çelik G, Ateş Ş, Erol E. Oscillator strengths and lifetimes for Cu I. Canadian Journal of Physics. 2015 Feb 11;93(10):1015-23.

[8] Çelik G, Atalay B, Ateş Ș. Radiative Lifetimes for Singly Ionized Beryllium. detail. 2016 Sep 1;15:20.

[9] Zheng N, Ma D, Yang R, Zhou T, Wang T, Han S. An efficient calculation of the energy levels of the carbon group. The Journal of Chemical Physics. 2000 Aug 1;113(5):1681-7.

[10] Zhang W, Palmeri P, Quinet P, Biémont E, Du S, Dai Z. Radiative-lifetime measurements and calculations of odd-parity highly excited levels in Ba I. Physical Review A. 2010 Oct 14;82(4):042507.

[11] Zhang W, Feng Y, Dai Z. Radiative lifetime measurements of odd-parity moderately excited levels belonging to $\mathrm{J}=0,1,2,3$ series in Sm I. JOSA B. 2010 Nov 1;27(11):2255-61.

[12] Çelik G, Erol E, Taşer M. Transition probabilities, oscillator strengths and radiative lifetimes for Zn II. Journal of Quantitative Spectroscopy and Radiative Transfer. 2013 Nov 1;129:263-71.

[13] Shizhong H, Qiufeng S. Calculation of the Rydberg Energy Levels for Francium Atom. Physics Research International. 2010 Dec 16;2010.

[14] Li S, Lei W, Hai-Feng Y, Xiao-Jun L, Hong-Ping L. Lifetime Measurement for 6snp Rydberg States of Barium. Chinese Physics Letters. 2011 Apr;28(4):043101.

[15] Glukhov IL, Nikitina EA, Ovsiannikov VD. Lifetimes of Rydberg states in ions of the group II elements. Optics and Spectroscopy. 2013 Jul 1;115(1):9-17.

[16] Hua J, Shi-Wei Y, Chang-Jian D. Lifetimes of Rydberg states of Eu atoms. Chinese Physics B. 2015 Jan;24(1):013203.

[17] Çelik G, Ateş Ş, Özarslan S, Taşer M. Transition probabilities, oscillator strengths and lifetimes for singly ionized magnesium. Journal of Quantitative Spectroscopy and Radiative Transfer. 2011 Sep 1;112(14):2330-

[18] Deller A, Alonso AM, Cooper BS, Hogan SD, Cassidy DB. Measurement of Rydberg positronium fluorescence lifetimes. Physical Review A. 2016 Jun 29;93(6):062513.

[19] Zheng N, Ma D, Yang R, Zhou T, Wang T, Han S. An efficient calculation of the energy levels of the carbon group. The Journal of Chemical Physics. 2000 Aug 1;113(5):1681-7.

[20] Zhang T, Zheng N, Ma D. Theoretical calculation of energy levels of Sr I. Physica Scripta. 2007 May 4;75(6):763.

[21] ZHOU C, CAO JJ, LIANG L, ZHANG L. Theoretical calculation of energy levels of Pb III. Turkish Journal of Physics. 2011 Apr 12;35(1):37-42.

[22] Zheng NW, Zhou T, Yang R, Wang T, Ma D. Analysis of the bound odd-parity spectrum of krypton by weakest bound electron potential model theory. Chemical Physics. 2000 Aug 1;258(1):37-46.

[23] Kramida, A., Ralchenko, Yu., Reader, J., and NIST ASD Team (2018). NIST Atomic Spectra Database (ver. 5.6.1), [Online]. Available: https://physics.nist.gov/asd [2019, March 21]. National Institute of Standards and Technology, Gaithersburg, MD. DOI: https://doi.org/10.18434/T4W30F [24] Zheng N, Ma D, Yang R, Zhou T, Wang T, Han S. An efficient calculation of the energy levels of the carbon group. The Journal of Chemical Physics. 2000 Aug 1;113(5):1681-7.

[25] Martin WC. Series formulas for the spectrum of atomic sodium (Na I). JOSA. 1980 Jul 1;70(7):7848.

[26] Langer R M 1930 A generalization of the Rydberg formula Phys. Rev. 35649768

[27] Verolaīnen YF, Nikolaich AY. Radiative lifetimes of excited states of atoms. Soviet Physics Uspekhi. 1982;25(6):431.

[28] https://en.wikipedia.org/wiki/Spectral_line

\section{Acknowledgement:}

This work is supported by Dr. Zaheer Uddin from University of Karachi, Department of Physics. We all are highly great full to Dr. Zaheer Uddin for providing the assistance during the completion of this work. 